\title{
Galerkin-Vlasov Method for the Flexural Analysis of Rectangular Kirchhoff Plates with Clamped and Simply Supported Edges
}

\author{
Nwoji, C.U. ${ }^{1}$, Mama, B.O. ${ }^{2}$, Ike, C. C. ${ }^{* 3}$, Onah, H.N. ${ }^{4}$ \\ ${ }^{I}$ Dept of Civil Engineering, University of Nigeria, Nsukka, Enugu State, Nigeria. \\ ${ }^{2}$ Lecturer, Dept of Civil Engineering, University of Nigeria, Nsukka, Enugu State, Nigeria. \\ ${ }^{3}$ Lecturer, Dept of Civil Engineering, Enugu State University of Science \& Technology, Enugu State, Nigeria. \\ ${ }^{4}$ Lecturer, Dept of Civil Engineering, University of Nigeria, Nsukka, Enugu State, Nigeria.
}

\begin{abstract}
This paper presents the flexural analysis using the Galerkin-Vlasov method for rectangular Kirchhoff plates with two opposite edges $(y= \pm b)$ clamped and the other two opposite edges $(x=0, x=2 a)$ simply supported. The plate considered is assumed homogeneous and isotropic and submitted to a uniformly distributed load, $p_{0}$ over the entire plate domain. The plate variational functional is constructed using the Galerkin-Vlasov philosophy by choosing the displacement shape functions to be a product of the eigen functions of a clamped - clamped Euler-Bernoulli beam in the corresponding clamped directions and the eigen function of a simply supported Euler - Bernoulli beam in the x-coordinate direction. The plate variational functional was then solved analytically to obtain the plate deflection function in terms of the spatial coordinates $(x, y)$. Maximum plate deflection and maximum bending moments were evaluated and found to occur at the plate centre. The maximum deflection and maximum bending moments were compared with solutions obtained by Timoshenko and Woinowsky-Krieger, and found to be in good agreement with an average relative error of $0.37 \%$ for a four term displacement shape function. Convergence to the exact Timoshenko solution is obtained using more terms in the displacement shape function. The bending moment distributions were also determined using the moment-displacement relations, and found to agree with solutions obtained by Timoshenko and Woinowsky-Krieger.
\end{abstract}

Keywords: Galerkin-Vlasov method, plate variational functional, eigenfunction, Kirchhoff plate, EulerBernoulli beam.

\section{Introduction}

Plates are three dimensional structural elements, having thicknesses much smaller than the other dimensions. The stress state in a typical plate problem is thus a three dimensional stress state; described using three dimensional theory of elasticity [1]. The behaviour of plates depends greatly on the ratio of the thickness to the least lateral dimension. Thus plates have been classified into three groups $[2,3]$ namely (i) thin plates with small deflections (ii) thin plates with large deflections and (iii) thick plates. Thin plates are defined as those plates which the ratio of the thickness to the smaller span length is less than 1/20 [2]. Ventsel and Krauthammar [4] define thin plates as plates with $a / h$ ratio in the range $8 \ldots 10 \leq a / h \leq 80 \ldots 10$

Plates are important structural components/elements extensively used in various engineering applications such as floor slabs, bridge decks, rigid pavements of highways and airport runways, ship decks, aerocraft and spacecraft panels and retaining walls. The behaviour of plates depends on the type and nature of load application, and in general, plates can be subject to flexural, dynamic, and buckling behaviours. Plates are also classified by their shapes and materials as rectangular, circular, elliptical, polygonal, orthotropic, anisotropic, isotropic, homogeneous and non-homogeneous (heterogeneous). In this work, the static flexural analysis of rectangular thin plates of the Kirchhoff plate model is performed using the Galerkin-Vlasov method.

\section{Literature Review}

Literature survey shows that several theories are used to describe the static flexure of plates. Szilard [3] presented the variable thickness thin plate theory as

$$
\nabla^{2}\left(D(x, y) \nabla^{2} w\right)-(1-\mu) L(D, w)=q_{z}(x, y)
$$

where $L(D, w)$ is a partial differential operator given by 


$$
L(D, w)=\frac{\partial^{2} D}{\partial x^{2}} \frac{\partial^{2} w}{\partial y^{2}}-2 \frac{\partial^{2} D}{\partial x \partial y} \frac{\partial^{2} w}{\partial x \partial y}+\frac{\partial^{2} D}{\partial y^{2}} \frac{\partial^{2} w}{\partial x^{2}}
$$

$D(x, y)$ is the flexural rigidity of the plate which is shown to vary with the spatial coordinates variables, $x$ and $y$, $\mu$ is the Poisson's ratio, $q_{z}(x, y)$ is the distributed transverse load on the plate, $w(x, y)$ is the plate transverse deflection.

Only a few problems of plates with variable flexural rigidity have been solved by the classical methods of Levy and Navier [4]. The complexity of the analysis of thin plates with variable thickness depends significantly on the mathematical expressions describing the thickness variation and the flexural rigidity [3]. Large deflection thin plate theory, originally formulated by von-Karman in 1910, comprise the following equilibrium and compatibility equations

$$
\begin{aligned}
& \frac{D}{h} \nabla^{4} w(x, y)=L(w, \phi)+\frac{p_{z}}{h} \\
& \frac{1}{E} \nabla^{4} \phi(x, y)=\frac{1}{2} L(w, w)
\end{aligned}
$$

where $L$ is a partial differential operator, defined as Equation (2), $E$ is the Young's modulus, $\phi$ is the Airy stress function, $h$ is the plate thickness, $p_{z}$ is the distributed load.

Von - Karman's large deflection thin plate equations are coupled non linear partial differential equations of fourth order in $w$ and $\phi(x, y)$.

Reissner's stress-based theory of plates, formulated by applying Castigliano's theorem of least work to a differential plate element, and using stresses as the unknown variables, yielded the following three simultaneous partial differential equations [5],

$$
\begin{aligned}
& D \nabla^{2} \nabla^{2} w(x, y)=p_{z}-\frac{h^{2}}{10}\left(\frac{2-\mu}{1-\mu}\right) \nabla^{2} p_{z} \\
& q_{x}=-\frac{D \partial \nabla^{2} w}{\partial x}+\frac{h^{2}}{10} \nabla^{2} q_{x}-\frac{h^{2}}{10}\left(\frac{1}{1-\mu}\right) \frac{\partial p_{z}}{\partial x} \\
& q_{y}=-D \frac{\partial}{\partial y} \nabla^{2} w+\frac{h^{2}}{10} \nabla^{2} q_{y}-\frac{h^{2}}{10}\left(\frac{1}{1-\mu}\right) \frac{\partial p_{z}}{\partial y}
\end{aligned}
$$

where $\nabla^{2}$ is the Laplacian operator in Cartesian coordinates.

Reissner's plate theory is an extension of the Kirchhoff - Love plate theory incorporating shear deformation effects; and is useful in moderately thick plates. Reissner's assumption of quadratic variation of shear stress through the thickness is consistent with theory of elasticity solution for plates under static flexure.

Mindlin relaxed Kirchhoff's hypothesis and using a displacement based approach, modified the Kirchhoff - Love plate theory, thus extending the scope of application to thick plates. Mindlin's first order shear deformation plate theory has been developed by Mindlin [6], Mindlin and Deresiewicz [7] and Mindlin et al [8]. They considered shear deformation effects on the plate flexural behaviour by assuming linear variations for all three displacement components across the plate thickness.

The governing partial differential equations (PDE) of isotropic Mindlin plates are a system of three partial differential equations in terms of three unknown displacement parameters, $w, \theta_{x}$ and $\theta_{y}$. They are [6,3]

$$
\begin{aligned}
& \nabla^{2} w+\frac{\partial \theta_{x}}{\partial x}+\frac{\partial \theta_{y}}{\partial y}=\frac{-q}{k G h} \\
& \nabla^{2}\left(\frac{\partial \theta_{x}}{\partial x}+\frac{\partial \theta_{y}}{\partial y}\right)=\frac{-q}{D} \\
& D\left(\frac{\partial^{2} \theta_{x}}{\partial x^{2}}+\mu \frac{\partial^{2} \theta_{y}}{\partial x \partial y}\right)+\left(\frac{1-\mu}{2}\right) D\left(\frac{\partial^{2} \theta_{x}}{\partial y^{2}}+\frac{\partial^{2} \theta_{y}}{\partial y \partial x}\right)=k G h\left(\theta_{x}+\frac{\partial w}{\partial x}\right)
\end{aligned}
$$




$$
D\left(\frac{\partial^{2} \theta_{y}}{\partial y^{2}}+\mu \frac{\partial^{2} \theta_{x}}{\partial x \partial y}\right)+\frac{(1-\mu) D}{2}\left(\frac{\partial^{2} \theta_{x}}{\partial x \partial y}+\frac{\partial^{2} \theta_{y}}{\partial x^{2}}\right)=k G h\left(\theta_{y}+\frac{\partial w}{\partial y}\right)
$$

where $k=$ shear correction factor

$G=$ shear modulus

$D=$ flexural rigidity of plate

$h=$ plate thickness

$q=$ transverse load

$\mu=$ Poisson's ratio of plate material

$\theta_{x}, \theta_{y}=$ rotations of plate middle surface

$w(x, y)=$ transverse displacement

Mindlin plate theory assumes the shear strain (shear stress) is constant across the plate thickness in violation of the predictions of the theory of elasticity solutions for plate flexure since the shear stress are known to have a parabolic (quadratic) variation over the plate thickness. Mindlin introduced a shear correction factor, $k$ to ensure the lowest amount of internal energy is predicted by the Mindlin theory. The shear stress correction factor, $k$, merely results in the resultant shear stress being in agreement with the theory of elasticity solutions, but the distribution of the shear stress over the plate thickness violate theory of elasticity solutions.

\section{Higher Order Shear Deformation Plate Theory (HSDPT)}

Chandrashekhara [9] developed a HSDPT for rectangular isotropic plates in flexure by limiting the displacement variation in the $x$ and $y$ coordinate directions to the cubic term of the thickness coordinate, $z$ while the lateral deflection in the $z$ direction is considered to vary only with the $x$ and $y$ coordinates. Using those assumptions on the displacement components, and the strain-displacement equations, the stress-strain law and equilibrium, the governing partial differential equations of the HSDPT were given by Chandrashekhara [9] as follows

$$
\begin{gathered}
\left(\frac{\partial^{3} w_{0}}{\partial x^{3}}+\frac{\partial^{3} w_{0}}{\partial x \partial y^{2}}\right)-\frac{17}{4}\left(\frac{\partial^{2} \psi_{x}}{\partial x^{2}}+\left(\frac{1-\mu}{2}\right) \frac{\partial^{2} \psi_{x}}{\partial y^{2}}+\left(\frac{1+\mu}{2}\right) \frac{\partial^{2} \psi_{y}}{\partial x \partial y}\right) \\
+\frac{21}{h^{2}}(1-\mu)\left(\psi_{x}+\frac{\partial w_{0}}{\partial x}\right)=0 \\
\left(\frac{\partial^{3} w_{0}}{\partial y^{3}}+\frac{\partial^{3} w_{0}}{\partial y \partial x^{2}}\right)-\frac{17}{4}\left(\frac{\partial^{2} \psi_{y}}{\partial y^{2}}+\left(\frac{1-\mu}{2}\right) \frac{\partial^{2} \psi_{y}}{\partial x^{2}}+\left(\frac{1+\mu}{2}\right) \frac{\partial^{2} \psi_{x}}{\partial x \partial y}\right) \\
+\frac{21}{h^{2}}(1-\mu)\left(\psi_{y}+\frac{\partial w_{0}}{\partial y}\right)=0 \\
\nabla^{4} w_{0}-\frac{16}{5}\left(\frac{\partial^{3} \psi_{x}}{\partial x^{3}}+\frac{\partial^{3} \psi_{x}}{\partial x \partial y^{2}}+\frac{\partial^{3} \psi_{y}}{\partial x^{2} \partial y}+\frac{\partial^{3} \psi_{y}}{\partial y^{3}}\right)-\frac{336}{5 h^{2}}(1-\mu) \nabla^{2} w_{0} \\
-\frac{336}{5 h^{2}}(1-\mu)\left(\frac{\partial \psi_{x}}{\partial x}+\frac{\partial \psi_{y}}{\partial y}\right)-\frac{21 q}{D}=0
\end{gathered}
$$

where $\psi_{x}, \psi_{y}$ are unknown parameters used to describe the displacement field. $w_{0}$ is the transverse deflection $q$ is the distributed transverse load. $D$ is the flexural rigidity of plate and $h$ is the plate thickness.

As is evident in the complex and complicated nature of the governing PDE, higher order plate theories are cumbersome and conceptually more demanding, because with each additional power of the thickness coordinate, more unknown variables are introduced into the governing equation, and some of these unknown variables may not have physical interpretation.

\section{Kirchhoff - Love Plate Theory (Classical Plate Theory)}

Kirchhoff plate theory is a linear plate theory that uncouples flexure and stretching behaviours of the plate. Kirchhoff plate theory reduces the three dimensional plate problem to a two dimensional problem by integrating out the thickness dimension and expressing equilibrium in terms of force resultants. The CPT is based in the following kinematic assumptions: 
(i) straight lines normal to the plate middle surface remain straight and normal to the middle surface after deformation (normality condition).

(ii) the thickness of the plate does not change during a deformation.

The governing partial differential equation for plate flexure is the fourth order PDE

$$
D \nabla^{4} w(x, y)=p_{z}(x, y)
$$

where $D=$ flexural rigidity of the plate $=\frac{E h^{3}}{12\left(1-\mu^{2}\right)}$

$w(x, y)=$ transverse displacement

$p_{z}(x, y)=$ transverse load on plate

In most plate problems, the plate thickness is small as compared to the other planar dimensions and the plate deflections are usually small in comparison with the thickness of the plate [10]. This makes the CPT the plate model of choice in the description of most of such plate problems where the plate is thin and the plate deformations are small compared with the plate thickness.

Some advantages of the CPT include [10],

(i) the bending and stretching behaviours are uncoupled but can be analysed together by linear addition due to the linear nature of the PDE.

(ii) the governing PDE involves only one unknown variable - the transverse displacement.

(iii) the stresses can be calculated from the stress displacement equations.

(iv) CPT yields parabolic variation of stress $\tau_{y z}$ and $\tau_{z x}$ over the thickness in agreement with structural analysis results.

\section{Methods for solving plate problems}

A review of literature shows that methods for solving plate problems can be classified into two broad groups namely: analytical methods, and numerical methods. Analytical methods aim at solving the governing equations of the plate problem in a mathematically rigorous manner, and thus to obtain solutions which exactly satisfy the governing equations within the plate domain as well as the plate boundaries. Analytical methods thus lead to closed form solutions for the plate problems. Typical analytical methods include Navier's double trigonometric series method [11], Levy's single trigonometric series method [12]. Numerical methods are methods used to obtain approximate solutions to the plate problem. They are particularly useful when closed form solutions cannot be obtained by rigorous mathematical solution of the plate problem. Numerical methods include variational Ritz methods, variational Galerkin methods, weighted residual methods, finite Difference methods, finite Element methods, Boundary Element methods, and collocation methods.

Variational methods of Ritz and Galerkin have been applied to the analysis of plates by Osadebe et al [13], Aginam et al [14], Mbakogu and Pavlovic [15], Reddy [16], Balasubramanian [17], and Kantorovich and Krylov [18]. Ezeh et al [19] presented finite difference method of plate analysis. Closed form analytical solutions of the plate problem using the methods of Navier, Levy and the separation of variables method have been presented in Ventsel and Krauthammer [4], Szilard [3], Chandrashekhara [9], Ugural [2] and Timoshenko and Woinowsky-Krieger [10].

\section{Research Aim and Objectives}

The principal aim of this study is to apply the Galerkin-Vlasov method for analysis of rectangular Kirchhoff plates with two opposite edges $x=0, x=2 a$ simply supported and the other two opposite edges $y=-b, \quad y=b$ clamped, for the case of uniformly distributed load. The specific objectives include:

(i) to present the Galerkin-Vlasov variational integral for the Kirchhoff plate with two opposite edges $x=0, x$ $=2 a$ simply supported and the other two edges $y=-b, y=b$ clamped for the case of uniformly distributed load.

(ii) to obtain solutions for the deflection and bending moment for the Kirchhoff plates problem formulated in (i) above

(iii) to compare the maximum values of the deflection, and bending moments obtained in (ii) above with the solutions from literature.

\section{Galerkin-Vlasov Formulation and Solution}

We consider the rectangular Kirchhoff plate with two opposite edges simply supported and the other two opposite edges clamped. The plate is submitted to a uniformly distributed transverse load of constant intensity $p_{0}$ over the entire domain of the plate. The $x$ and $y$ coordinate axes are defined as shown on Figure 1. The plate domain is 
$0 \leq x \leq 2 a$
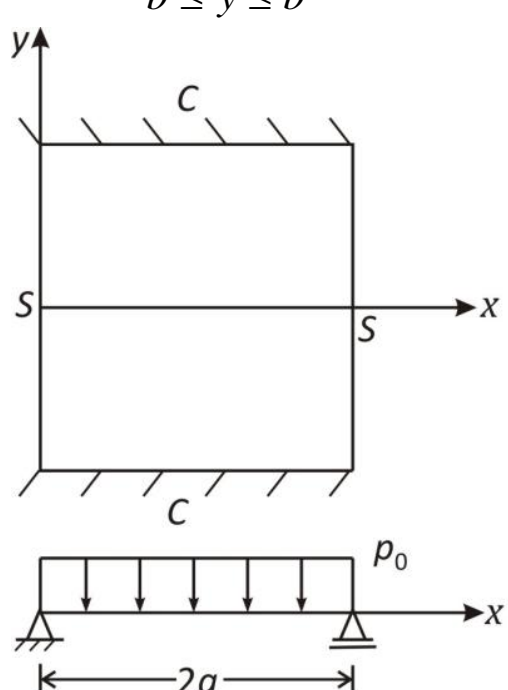

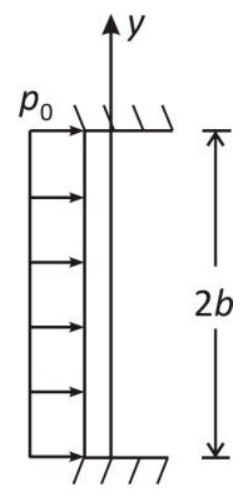

In the Galerkin-Vlasov method, the displacement shape functions are chosen to be the eigen-functions of a clamped Euler Bernoulli beam in the y-coordinate direction and the eigen-functions of a simply supported Euler Bernoulli beam in the $\mathrm{x}$-coordinate direction. Hence, the shape functions of the displacement field are given by

$$
\begin{aligned}
& F_{m}(x)=\sin \frac{m \pi x}{2 a} ; \quad 0 \leq x \leq 2 a, \quad m=1,3,5, \ldots \\
& G_{n}(y)=\frac{\cosh \frac{k_{n} y}{b}}{\cosh k_{n}}-\frac{\cos \frac{k_{n} y}{b}}{\cos k_{n}} ; \quad-b \leq y \leq b \quad n=1,2,3, \ldots
\end{aligned}
$$

where $k_{1}=2.36502 ; k_{2}=5.49280$

$$
\begin{aligned}
& k_{3}=8.63938 \quad k_{4}=11.78097 \\
& k_{n}=\frac{(4 n-1) \pi}{4} \text { for large values of } n .
\end{aligned}
$$

$k_{n}$ are the roots of the transcendental equation $\tan k_{n}+\tanh k_{n}=0$

The Galerkin-Vlasov variational functional is given by the double integral:

$$
\iint_{R}\left(D \nabla^{4} w-p(x, y)\right) \varphi_{i j}(x, y) d x d y=0
$$


where $\varphi_{i j}(x, y)$ are the shape functions of the displacement field, and $R$ the domain of the multiple integral is the two dimensional region of the plate domain.

Here,

$$
\varphi_{i j}(x, y)=F_{i}(x) G_{j}(y)
$$

Thus, we assume the displacement field as

$$
W(x, y)=\sum_{m}^{\infty} \sum_{n}^{\infty} W_{m n} F_{m}(x) G_{n}(y)
$$

where $W_{m n}$ are the displacement parameters we seek to determine.

We also express the distributed transverse load $p(x, y)$ in terms of the beam orthogonal basis functions $F_{m} G_{n}$ as

$$
p(x, y)=\sum_{m}^{\infty} \sum_{n}^{\infty} P_{m n} F_{m}(x) G_{n}(y)
$$

where $p_{m n}$ are the load coefficients of the orthogonal series expansions.

The Galerkin-Vlasov variational functional for the Kirchhoff plate problem becomes

$$
\begin{gathered}
\iint\left(\nabla^{4} \sum \sum W_{m n} F_{m}(x) G_{n}(y)-\sum \sum p_{m n} F_{m}(x) G_{n}(y)\right) F_{i}(x) G_{j}(y) d x d y=0 \\
\sum_{m}^{\infty} \sum_{n}^{\infty} W_{m n} \iint\left(\nabla^{4} F_{m}(x) G_{n}(y)\right) F_{i}(x) G_{j}(y) d x d y \\
=\sum_{m}^{\infty} \sum_{n}^{\infty} \frac{p_{m n}}{D} \iint\left(F_{m}(x) G_{n}(y)\right) F_{i}(x) G_{j}(y) d x d y
\end{gathered}
$$

The displacement shape functions of the Galerkin-Vlasov formulation presented in this study are orthogonal basis functions, and the above equations simplify to

$$
\begin{gathered}
\sum_{m}^{\infty} \sum_{n}^{\infty} w_{m n} \iint_{R}\left(F_{m}^{i v} F_{m} G_{m}^{2}+2 F_{m}^{\prime \prime} F_{m} G_{n} G_{n}^{\prime \prime}+G_{n}^{i v} G_{n} F_{m}^{2}\right) d x d y \\
=\sum_{m}^{\infty} \sum_{n}^{\infty} \frac{p_{m n}}{D} \iint_{R} F_{m}^{2} G_{n}^{2} d x d y
\end{gathered}
$$

Thus,

$$
\begin{aligned}
& w_{m n}=\frac{p_{m n} \iint_{R} F_{m}^{2} G_{n}^{2} d x d y}{D \iint_{R}\left(F_{m}^{i v} F_{m} G_{n}^{2}+2 F_{n}^{\prime \prime} F_{m} G_{n}^{\prime \prime} G_{n}+G_{n}^{i v} G_{n} F_{m}^{2}\right) d x d y} \\
& =\frac{p_{m n} \int F_{m}^{2}(x) d x \int G_{n}^{2}(y) d y}{D\left\{\int F_{m}^{i v} F_{m} d x \int G_{n}^{2} d y+2 \int F_{m}^{\prime \prime} F_{m} d x \int G_{n}^{\prime \prime} G_{n} d y+\int G_{n}^{i v} G_{n} d y \int F_{m}^{2} d x\right\}} \\
& w_{m n}=\frac{p_{m n} I_{2} I_{6}}{D\left(I_{1} I_{2}+2 I_{3} I_{4}+I_{5} I_{6}\right)}
\end{aligned}
$$

The integrations are evaluated using Mathematica software on line integrator. where

$$
\begin{array}{lll}
I_{1}=\int_{0}^{2 a} F_{m}^{i v} F_{m} d x & I_{2}=\int_{-b}^{b} G_{n}^{2} d y & I_{3}=\int_{0}^{2 a} F_{m}^{\prime \prime} F_{m} d x \\
I_{4}=\int_{-b}^{b} G_{n}^{\prime \prime} G_{n} d y & I_{5}=\int_{-b}^{b} G_{n}^{i v} G_{n} d y & I_{6}=\int_{0}^{2 a} F_{m}^{2} d x
\end{array}
$$

The integrals are evaluated using the online Mathematica integrator software to obtain 


$$
\begin{aligned}
& I_{1}=\left(\frac{m \pi}{2 a}\right)^{4} a \\
& I_{2}=2 b \\
& I_{3}=-\left(\frac{m \pi}{2 a}\right)^{2} a \\
& I_{4}=\frac{k_{n}}{b}\left[\frac{1}{\cosh ^{2} k_{n}}\left(\frac{\sinh 2 k_{n}}{2}+k_{n}\right)-\frac{1}{\cos ^{2} k_{n}}\left(\frac{\sin 2 k_{n}}{2}+k_{n}\right)\right] \\
& I_{5}=\left(\frac{k_{n}}{b}\right)^{4} 2 b \\
& I_{6}=a
\end{aligned}
$$

For the uniformly distributed transverse load $p(x, y)=p_{0}$, where $p_{0}$ is the intensity and

$$
\begin{aligned}
& p_{m n}=\frac{\int_{-b}^{b} \int_{0}^{2 a} p(x, y) F_{m} G_{n} d x d y}{\int_{-b}^{b} \int_{0}^{2 a} F_{m}^{2} G_{n}^{2} d x d y} \\
& p_{m n}=\frac{4 p_{0}}{m \pi k_{n}}\left(\tanh k_{n}-\tan k_{n}\right)
\end{aligned}
$$

Then,

$$
\begin{aligned}
& w_{m n}=\frac{\frac{4 p_{0}}{m \pi k_{n}}\left(\tanh k_{n}-\tan k_{n}\right) 2 a b}{D\left[\left(\frac{m \pi}{2 a}\right)^{4} 2 a b+2\left(-\left(\frac{m \pi}{2 a}\right)^{2} a\left(\frac{k_{n}}{b}\right)\right)\left[\frac{1}{\cosh ^{2} k_{n}}\left(\frac{\sinh 2 k_{n}}{2}+k_{n}\right)\right.\right.} \\
& \left.\left.-\frac{1}{\cos ^{2} k_{n}}\left(\frac{\sin 2 k_{n}}{2}+k_{n}\right)\right]+\left(\frac{k_{n}}{b}\right)^{4} 2 a b\right] \\
& \frac{8 p_{0}}{m \pi k_{n}}\left(\tanh k_{n}-\tan k_{n}\right) a b \\
& w_{m n}=\overline{D\left[\frac{m^{4} \pi^{4}}{8 a^{3}} b-\frac{m^{2} \pi^{2}}{2 a} \frac{k_{n}}{b}\left[\frac{1}{\cosh ^{2} k_{n}}\left(\frac{\sinh 2 k_{n}}{2}+k_{n}\right)-\frac{1}{\cos ^{2} k_{n}}\left(\frac{\sin 2 k_{n}}{2}+k_{n}\right)\right]+\frac{2 k_{n}^{4} a}{b^{3}}\right]} \\
& w_{m n}=\frac{\frac{8 p_{0}}{m \pi k_{n}}\left(\tanh k_{n}-\tan k_{n}\right) b^{2} \alpha}{D\left[\left(\frac{m^{4} \pi^{4}}{8 b^{2} \alpha^{3}}\right)-\left(\frac{m^{2} \pi^{2} k_{n}}{2 b^{2} \alpha}\right)\left[\frac{1}{\cosh ^{2} k_{n}}\left(\frac{\sinh 2 k_{n}}{2}+k_{n}\right)-\frac{1}{\cos ^{2} k_{n}}\left(\frac{\sin 2 k_{n}}{2}+k_{n}\right)\right]+\frac{2 k_{n}^{4} \alpha}{b^{2}}\right]} \\
& w_{m n}=\frac{\frac{8\left(\tanh k_{n}-\tan k_{n}\right)}{m \pi k_{n}} \frac{q_{0} b^{4} \alpha^{4}}{D}}{\left(\frac{m^{4} \pi^{4}}{8}+2 k_{n}^{4} \alpha^{4}-\frac{m^{2} \pi^{2}}{2} k_{n} \alpha^{2}\left[f\left(k_{n}\right)\right]\right)}
\end{aligned}
$$




$$
\begin{gathered}
w_{m n}=F(\alpha) \frac{q_{0} a^{4}}{D} \\
f\left(k_{n}\right)=\frac{1}{\cosh ^{2} k_{n}}\left(\frac{\sinh 2 k_{n}}{2}+k_{n}\right)-\frac{1}{\cos ^{2} k_{n}}\left(\frac{\sin 2 k_{n}}{2}+k_{n}\right) \\
F_{1}(\alpha)=\frac{8\left(\tanh k_{n}-\tan k_{n}\right)}{m \pi k_{n} G(\alpha)} \\
G_{1}(\alpha)=\frac{m^{4} \pi^{4}}{8}+2 k_{n}^{4} \alpha^{4}-\frac{m^{2} \pi^{2}}{2} k_{n} \alpha^{2} f\left(k_{n}\right) \\
f\left(k_{n}\right)=\frac{1}{\cosh ^{2} k_{n}}\left(\frac{\sinh 2 k_{n}}{2}+k_{n}\right)-\frac{1}{\cos ^{2} k_{n}}\left(\frac{\sin 2 k_{n}}{2}+k_{n}\right)
\end{gathered}
$$

The deflection thus becomes

$$
\begin{aligned}
& w(x, y)=\sum_{m}^{\infty} \sum_{n}^{\infty} w_{m n} \sin \frac{m \pi x}{2 a}\left(\frac{\cosh \frac{k_{n} y}{b}}{\cosh k_{n}}-\frac{\cos \frac{k_{n} y}{b}}{\cos k_{n}}\right) \\
& \text { for } m=1,3,5 \ldots \quad n=1,2,3,4, \ldots
\end{aligned}
$$

From considerations of the symmetrical nature of the plate and the symmetrical nature of the distributed transverse loading, the maximum deflection occurs at the centre of the plate $(x=a, y=0)$. Thus the maximum deflection is evaluated as

$$
\begin{aligned}
& w_{\max }=\sum_{m}^{\infty} \sum_{n}^{\infty} w_{m n} \sin \frac{m \pi}{2}\left(\frac{\cosh 0}{\cosh k_{n}}-\frac{\cos 0}{\cos k_{n}}\right)=w(x=a, y=0) \\
& m=1,3,5,7,9, \ldots \\
& w_{\max }=\sum_{m}^{\infty} \sum_{n}^{\infty} w_{m n}(-1)^{\frac{m-1}{2}}\left(\frac{1}{\cosh k_{n}}-\frac{1}{\cos k_{n}}\right)
\end{aligned}
$$

The values of the maximum deflection are evaluated for various values of the plate aspect ratios, and using a one term and four term truncation of the orthogonal series $F_{m}(x) G_{n}(y)$ and presented in Table 1 . These maximum deflection values are then compared with the classical solutions obtained by Timoshenko and Woinowsky-Krieger who solved the same problem by applying the principle of superposition.

\section{Bending Moment Distribution}

The bending moment distributions are given by

$$
\begin{aligned}
& M_{x x}=-D\left(\frac{\partial^{2} w}{\partial x^{2}}+\mu \frac{\partial^{2} w}{\partial y^{2}}\right) \\
& M_{y y}=-D\left(\frac{\partial^{2} w}{\partial y^{2}}+\mu \frac{\partial^{2} w}{\partial x^{2}}\right) \\
& \frac{\partial^{2} w}{\partial x^{2}}=-\sum_{m}^{\infty} \sum_{n}^{\infty} w_{m n}\left(\frac{m \pi}{2 a}\right)^{2} \sin \frac{m \pi x}{2 a}(G(y)) \\
& \frac{\partial^{2} w}{\partial y^{2}}=\sum_{m}^{\infty} \sum_{n}^{\infty} w_{m n} \sin \frac{m \pi x}{2 a}\left(G^{\prime \prime}(y)\right) \\
& G_{n}^{\prime}(y)=\left(\frac{k_{n}}{b}\right)\left(\frac{\sinh \frac{k_{n} y}{b}}{\cosh k_{n}}+\frac{\sin \frac{k_{n} y}{b}}{\cos k_{n}}\right)
\end{aligned}
$$




$$
\begin{aligned}
& G^{\prime \prime}(y)=\left(\frac{k_{n}}{b}\right)^{2}\left(\frac{\cosh \frac{k_{n} y}{b}}{\cosh k_{n}}+\frac{\cos \frac{k_{n} y}{b}}{\cos k_{n}}\right) \\
& \frac{\partial^{2} w}{\partial y^{2}}=\sum_{m}^{\infty} \sum_{n}^{\infty} w_{m n}\left(\frac{k_{n}}{b}\right)^{2} \sin \frac{m \pi x}{2 a}\left(\frac{\cosh \frac{k_{n} y}{b}}{\cosh k_{n}}+\frac{\cos \frac{k_{n} y}{b}}{\cos k_{n}}\right) \\
& M_{x x}=-D \sum_{m}^{\infty} \sum_{n}^{\infty}\left\{-w_{m n}\left(\frac{m \pi}{2 a}\right)^{2} \sin \frac{m \pi x}{2 a}\left(\frac{\cosh \frac{k_{n} y}{b}}{\cosh k_{n}}-\frac{\cos \frac{k_{n} y}{b}}{\cos k_{n}}\right)\right. \\
& \left.+\mu w_{m n}\left(\frac{k_{n}}{b}\right)^{2} \sin \frac{m \pi x}{2 a}\left(\frac{\cosh \frac{k_{n} y}{b}}{\cosh k_{n}}+\frac{\cos \frac{k_{n} y}{b}}{\cos k_{n}}\right)\right\} \\
& M_{x x}=-D \sum_{m}^{\infty} \sum_{n}^{\infty} w_{m n}\left\{-\left(\frac{m \pi}{2 a}\right)^{2} \sin \frac{m \pi x}{2 a}\left(\frac{\cosh \frac{k_{n} y}{b}}{\cosh k_{n}}-\frac{\cos \frac{k_{n} y}{b}}{\cos k_{n}}\right)\right. \\
& \left.+\mu\left(\frac{k_{n}}{b}\right)^{2} \sin \frac{m \pi x}{2 a}\left(\frac{\cosh \frac{k_{n} y}{b}}{\cosh k_{n}}+\frac{\cos \frac{k_{n} y}{b}}{\cos k_{n}}\right)\right\} \\
& M_{y y}=-D \sum_{m}^{\infty} \sum_{n}^{\infty} w_{m n}\left(\frac{k_{n}}{b}\right)^{2} \sin \frac{m \pi x}{2 a}\left(\frac{\cosh \frac{k_{n} y}{b}}{\cosh k_{n}}+\frac{\cos \frac{k_{n} y}{b}}{\cos k_{n}}\right) \\
& \left.-\mu w_{m n}\left(\frac{m \pi}{2 a}\right)^{2} \sin \frac{m \pi x}{2 a}\left(\frac{\cosh \frac{k_{n} y}{b}}{\cosh k_{n}}-\frac{\cos \frac{k_{n} y}{b}}{\cos k_{n}}\right)\right\}
\end{aligned}
$$

At the centre of the plate, $x=a, y=0$

$$
\begin{gathered}
M_{x x}(a, 0)=-D \sum_{m}^{\infty} \sum_{n}^{\infty}-w_{m n}\left(\frac{m \pi}{2 a}\right)^{2} \sin \frac{m \pi}{2}\left(\frac{1}{\cosh k_{n}}-\frac{1}{\cos k_{n}}\right) \\
\left.+\mu w_{m n}\left(\frac{k_{n}}{b}\right)^{2} \sin \frac{m \pi}{2}\left(\frac{1}{\cosh k_{n}}+\frac{1}{\cos k_{n}}\right)\right\} \\
=-D \sum_{m}^{\infty} \sum_{n}^{\infty}-w_{m n}\left\{\left(\frac{m \pi}{2 a}\right)^{2}\left(\frac{1}{\cosh k_{n}}-\frac{1}{\cos k_{n}}\right)\right. \\
\left.-\mu\left(\frac{k_{n}}{b}\right)^{2}\left(\frac{1}{\cosh k_{n}}+\frac{1}{\cos k_{n}}\right)\right\}(-1)^{\frac{m-1}{2}}
\end{gathered}
$$




$$
\begin{aligned}
=-D \sum_{m}^{\infty} \sum_{n}^{\infty} \frac{F_{1}(\alpha) p_{0} a^{4}}{D}\left\{\left(\frac{m \pi}{2 a}\right)^{2}\left(\frac{-1}{\cosh k_{n}}+\frac{1}{\cos k_{n}}\right)\right. \\
\left.+\mu\left(\frac{k_{n}}{b}\right)^{2}\left(\frac{1}{\cosh k_{n}}+\frac{1}{\cos k_{n}}\right)\right\}(-1)^{\frac{m-1}{2}} \\
M_{x x}=-\sum_{m}^{\infty} \sum_{n}^{\infty} F_{1}(\alpha) p_{0} a^{4}\left\{\left(\frac{m \pi}{2 a}\right)^{2}\left(\frac{1}{\cos k_{n}}-\frac{1}{\cosh k_{n}}\right)\right. \\
\left.+\mu\left(\frac{k_{n}}{b}\right)^{2}\left(\frac{1}{\cosh k_{n}}+\frac{1}{\cos k_{n}}\right)\right\}(-1)^{\frac{m-1}{2}}
\end{aligned}
$$

Similarly, at the centre,

$$
\begin{gathered}
M_{y y}(a, 0)=-D \sum_{m}^{\infty} \sum_{n}^{\infty} w_{m n}\left(\frac{k_{n}}{b}\right)^{2} \sin \frac{m \pi}{2}\left(\frac{1}{\cosh k_{n}}+\frac{1}{\cos k_{n}}\right) \\
\left.-\mu w_{m n}\left(\frac{m \pi}{2 a}\right)^{2} \sin \frac{m \pi}{2}\left(\frac{1}{\cosh k_{n}}-\frac{1}{\cos k_{n}}\right)\right\} \\
=-D \sum_{m}^{\infty} \sum_{n}^{\infty} \frac{F_{1}(\alpha) p_{0} a^{4}}{D}\left\{\left(\frac{k_{n}}{b}\right)^{2}\left(\frac{1}{\cosh k_{n}}+\frac{1}{\cos k_{n}}\right)\right. \\
\left.-\mu\left(\frac{m \pi}{2 a}\right)^{2}\left(\frac{1}{\cosh k_{n}}-\frac{1}{\cos k_{n}}\right)\right\}(-1)^{\frac{m-1}{2}}
\end{gathered}
$$

The bending moment at the middle of the clamped edge $(x=a, y= \pm b)$ is

$$
\begin{aligned}
& M_{x x}(a, y= \pm b)=-D \sum_{m}^{\infty} \sum_{n}^{\infty} w_{m n}\left\{-\left(\frac{m \pi}{2 a}\right)^{2} \sin \frac{m \pi}{2}\left(\frac{\cosh k_{n}}{\cosh k_{n}}-\frac{\cos k_{n}}{\cos k_{n}}\right)\right. \\
&\left.+\mu\left(\frac{k_{n}}{b}\right)^{2} \sin \frac{m \pi}{2}\left(\frac{\cosh k_{n}}{\cosh k_{n}}+\frac{\cos k_{n}}{\cos k_{n}}\right)\right\} \\
& M_{x x}(a, y= \pm b)=-D \sum_{m}^{\infty} \sum_{n}^{\infty} w_{m n} \cdot \mu\left(\frac{k_{n}}{b}\right)^{2} 2(-1)^{\frac{m-1}{2}}
\end{aligned}
$$

Similarly,

$$
\begin{aligned}
M_{y y}(a, y= \pm b) & =-D \sum_{m}^{\infty} \sum_{n}^{\infty} w_{m n}\left\{\left(\frac{k_{n}}{b}\right)^{2}\left(\frac{\cosh k_{n}}{\cosh k_{n}}+\frac{\cos k_{n}}{\cos k_{n}}\right)\right. \\
& \left.-\mu\left(\frac{m \pi}{2 a}\right)^{2}\left(\frac{\cosh k_{n}}{\cosh k_{n}}-\frac{\cos k_{n}}{\cos k_{n}}\right)\right\}(-1)^{\frac{m-1}{2}} \\
= & -D \sum_{m}^{\infty} \sum_{n}^{\infty} w_{m n}\left(\frac{k_{n}}{b}\right)^{2} 2(-1)^{\frac{m-1}{2}}
\end{aligned}
$$

The bending moments expressions obtained for $M_{x x}, M_{y y}$ are computed at the centre of the plate and at the middle of the clamped edge and shown in tabulated form in Tables 6,7 and 8.

Timoshenko and Woinowsky-Krieger [10] obtained their solutions for the same problem of rectangular Kirchhoff plate with two opposite edges $(x=0, x=2 a)$ simply supported and the other edges $y= \pm b$ clamped by first solving the problem using the assumption that all the four edges are simply supported, and then applying bending moments along the clamped edges by the applied distributed transverse load. Timoshenko and 
Galerkin-Vlasov Method for the Flexural Analysis of Rectangular Kirchhoff Plates with Clamped ..

Woinowsky-Krieger's solution were adapted to the same problem of plates, simply supported at $x=0, x=2 a$ and clamped at $y= \pm b$, and then compared with the solutions in this study. The comparison of the present results and the results by Timoshenko and Woinowsky-Krieger are presented in Tables 1, 2, 3, 6, 7 and 8 . Convergence studies are also presented in Tables 4 and 5.

Table 1: Maximum deflection of SCSC thin plate $w_{\max }=F_{m n}(\alpha)\left(\frac{p b^{4}}{D}\right)$ $(b<a)$

\begin{tabular}{|l|l|l|l|l|l|}
\hline$a / b$ & $\begin{array}{l}\text { Present study } m=1, \\
n=1, F_{m n}(\alpha)\end{array}$ & $\begin{array}{l}\text { Relative } \\
\text { error } \%\end{array}$ & $\begin{array}{l}\text { Present study } m=1,3, \\
n=1,2 F_{m n}(\alpha)\end{array}$ & $\begin{array}{l}\text { Relative } \\
\text { error } \%\end{array}$ & $\begin{array}{l}\text { Timoshenko and Woinowsky- } \\
\text { Krieger [10] }\end{array}$ \\
\hline 1.0 & & & & & \\
\hline 1.1 & 0.03501 & 4.69 & 0.03331 & -0.39 & 0.03344 \\
\hline 1.2 & 0.03753 & 5.18 & 0.03546 & -0.62 & 0.03568 \\
\hline 1.3 & 0.03963 & 5.85 & 0.03713 & -0.83 & 0.03744 \\
\hline 1.4 & 0.04137 & 7.73 & 0.03839 & -0.03 & 0.03840 \\
\hline 1.5 & 0.04282 & 8.35 & 0.03939 & -0.45 & 0.03952 \\
\hline 2 & 0.04238 & 1.875 & 0.04150 & -0.24 & 0.04160 \\
\hline$\infty$ & 0.04218 & 1.39 & 0.04162 & -0.05 & 0.04160 \\
\hline
\end{tabular}

Table 2: Maximum deflection coefficients of Kirchhoff plate with two opposite edges $(x=0, x=2 a)$ simply supported and two opposite edges $(y=-b, y=b)$ clamped and subject to uniformly distributed load $(b<a)$

\begin{tabular}{|l|l|l|}
\hline \multirow{2}{*}{$a / b$} & \multicolumn{2}{|c|}{$w_{\max }=F(\alpha) p_{0} b^{4} / D$} \\
\cline { 2 - 3 } & Present study \\
\hline 1.1 & 0.03344 & Timoshenko and Woinowsky-Krieger [10] \\
\hline 1.2 & 0.03568 & 0.03344 \\
\hline 1.3 & 0.03744 & 0.03568 \\
\hline 1.4 & 0.03840 & 0.03744 \\
\hline 1.5 & 0.03952 & 0.03840 \\
\hline 2 & 0.04160 & 0.03952 \\
\hline$\infty$ & 0.04160 & 0.04160 \\
\hline
\end{tabular}

Table 3: Coefficients for Maximum Deflection for Kirchhoff plate with two opposite edges $(x=0, x=2 a)$ simply supported and two opposite edges $(y=-b, y=b)$ clamped, and under uniformly distributed load $p_{0}$ $(b>a)$

\begin{tabular}{|l|l|l|}
\hline \multirow{3}{*}{$b / a$} & \multicolumn{2}{|c|}{$w_{\max }=F_{m m}(\alpha) p_{0} a^{4} / D$} \\
\cline { 2 - 3 } & Present study & Timoshenko and Woinowsky-Krieger [10] \\
\hline 1 & 0.03072 & 0.03072 \\
\hline 1.1 & 0.04015 & 0.04015 \\
\hline 1.2 & 0.05014 & 0.05014 \\
\hline 1.3 & 0.06208 & 0.06208 \\
\hline 1.4 & 0.07360 & 0.07360 \\
\hline 1.5 & 0.08496 & 0.08496 \\
\hline 1.6 & 0.09648 & 0.09648 \\
\hline 1.7 & 0.10688 & 0.10688 \\
\hline 1.8 & 0.11712 & 0.11712 \\
\hline 1.9 & 0.12640 & 0.12640 \\
\hline 2 & 0.13504 & 0.13504 \\
\hline 3 & 0.18688 & 0.18688 \\
\hline$\infty$ & 0.20832 & 0.20832 \\
\hline
\end{tabular}

Table 4: Convergence study of the maximum deflection function (for $b<a$ )

\begin{tabular}{|l|l|l|l|l|l|}
\hline$a / b$ & $m=1$ & $m=1,3$ & $m=1,3,5$ & $m=1,3,5,7$ & $m=1,3,5,7,9$ \\
$n=1$ & $n=1,2$ & $n=1,2,3$ & $n=1,2,3,4$ & $n=1,2,3,4,5$ \\
\hline 1.1 & 0.03501 & 0.03331 & 0.03346 & 0.03343 & 0.03344 \\
\hline 1.2 & 0.03753 & 0.03546 & 0.03565 & 0.03561 & 0.03568 \\
\hline 1.3 & 0.03963 & 0.03713 & 0.03737 & 0.03732 & 0.03744 \\
\hline 1.4 & 0.04137 & 0.03839 & 0.03869 & 0.03863 & 0.03840 \\
\hline 1.5 & 0.04282 & 0.03934 & 0.03971 & 0.03963 & 0.03952 \\
\hline 2 & 0.04238 & 0.04105 & 0.04197 & 0.04177 & 0.04160 \\
\hline
\end{tabular}


Galerkin-Vlasov Method for the Flexural Analysis of Rectangular Kirchhoff Plates with Clamped ..

\begin{tabular}{|l|l|l|l|l|l|l|}
\hline & 0.04218 & 0.04162 & 0.04168 & 0.04166 & 0.04160 \\
\hline
\end{tabular}
\begin{tabular}{|l|l|l|l|l|l|}
\hline$a / b$ & $\begin{array}{l}m=1 \\
n=1\end{array}$ & $\begin{array}{l}m=1,3 \\
n=1,2\end{array}$ & $\begin{array}{l}m=1,3,5 \\
n=1,2,3\end{array}$ & $\begin{array}{l}m=1,3,5,7 \\
n=1,2,3,4\end{array}$ & $\begin{array}{l}m=1,3,5,7,9 \\
n=1,2,3,4,5\end{array}$ \\
\hline 1 & 0.03197 & 0.03058 & 0.03071 & 0.03068 & 0.03072 \\
\hline 1.1 & 0.04200 & 0.04033 & 0.04048 & 0.04044 & 0.04015 \\
\hline 1.2 & 0.05297 & 0.05095 & 0.05113 & 0.05109 & 0.05014 \\
\hline 1.3 & 0.06456 & 0.06212 & 0.06235 & 0.06230 & 0.06208 \\
\hline 1.4 & 0.07645 & 0.07351 & 0.07280 & 0.07374 & 0.07360 \\
\hline 1.5 & 0.08836 & 0.08484 & 0.08520 & 0.08512 & 0.08496 \\
\hline 1.6 & 0.10006 & 0.09588 & 0.09632 & 0.09622 & 0.09648 \\
\hline 1.7 & 0.11137 & 0.10643 & 0.10697 & 0.10689 & 0.10688 \\
\hline 1.8 & 0.12216 & 0.11638 & 0.11703 & 0.11689 & 0.11712 \\
\hline 1.9 & 0.13236 & 0.12564 & 0.12643 & 0.12626 & 0.12640 \\
\hline 2 & 0.14192 & 0.13418 & 0.13512 & 0.13492 & 0.13504 \\
\hline 3 & 0.20587 & 0.18406 & 0.18772 & 0.18682 & 0.18688 \\
\hline$\infty$ & 0.20914 & 0.20828 & 0.20834 & 0.20833 & 0.20832 \\
\hline
\end{tabular}

Table 6: Bending moment coefficients for Kirchhoff plate with two opposite edges $(x=0, x=2 a)$ simply supported, and two opposite edges $(y= \pm b)$ clamped.

$\mu=0.30 \quad(b<a)$

\begin{tabular}{|l|l|l|l|l|}
\hline \multirow{3}{*}{$a / b$} & \multicolumn{2}{|l|}{$M_{x x_{c}}=\beta_{1} p_{0} b^{2}$} & \multicolumn{2}{l|}{$M_{y y_{c}}=\beta_{2} p_{0} b^{2}$} \\
\cline { 2 - 5 } & Present study & $\begin{array}{l}\text { Timoshenko and Woinowsky-Krieger } \\
{[10]}\end{array}$ & Present study & $\begin{array}{l}\text { Timoshenko and Woinowsky-Krieger } \\
{[10]}\end{array}$ \\
\hline 1.1 & 0.092 & 0.092 & 0.142 & 0.142 \\
\hline 1.2 & 0.086 & 0.086 & 0.150 & 0.150 \\
\hline 1.3 & 0.0812 & 0.0812 & 0.1552 & 0.1552 \\
\hline 1.4 & 0.0768 & 0.0768 & 0.1596 & 0.1596 \\
\hline 1.5 & 0.0716 & 0.0716 & 0.1624 & 0.1624 \\
\hline 2 & 0.0568 & 0.0568 & 0.168 & 0.168 \\
\hline$\infty$ & 0.05 & 0.05 & 0.1668 & 0.1668 \\
\hline
\end{tabular}

Table 7: Bending moment coefficients for Kirchhoff plate with two opposite edges $(x=0, x=2 a)$ simply supported and two opposite edges $(y= \pm b)$ clamped.

$\mu=0.30 \quad(b<a)$

\begin{tabular}{|l|l|l|l|l|}
\hline \multirow{3}{*}{$b / a$} & \multicolumn{2}{|l|}{$M_{x x}=\beta_{1} p_{0} a^{2}$} & \multicolumn{2}{l|}{$M_{y y}=\beta_{2} p_{0} a^{2}$} \\
\cline { 2 - 5 } & Present study & $\begin{array}{l}\text { Timoshenko and Woinowsky-Krieger } \\
{[10]}\end{array}$ & Present study & $\begin{array}{l}\text { Timoshenko and Woinowsky-Krieger } \\
{[10]}\end{array}$ \\
\hline 1. & 0.0976 & 0.0976 & 0.1328 & 0.1328 \\
\hline 1.1 & 0.1228 & 0.1228 & 0.1484 & 0.1484 \\
\hline 1.2 & 0.1504 & 0.1504 & 0.16 & 0.16 \\
\hline 1.3 & 0.1784 & 0.1784 & 0.1704 & 0.1704 \\
\hline 1.4 & 0.2056 & 0.2056 & 0.1792 & 0.1792 \\
\hline 1.5 & 0.234 & 0.234 & 0.184 & 0.184 \\
\hline 1.6 & 0.260 & 0.260 & 0.1876 & 0.1876 \\
\hline 1.7 & 0.2848 & 0.2848 & 0.190 & 0.190 \\
\hline 1.8 & 0.3072 & 0.3072 & 0.1908 & 0.1908 \\
\hline 1.9 & 0.3284 & 0.3284 & 0.1904 & 0.1904 \\
\hline 2 & 0.3476 & 0.3476 & 0.1896 & 0.1896 \\
\hline 3 & 0.4576 & 0.4576 & 0.1676 & 0.1676 \\
\hline$\infty$ & 0.050 & 0.050 & 0.150 & 0.150 \\
\hline
\end{tabular}

Table 8: Bending moment coefficients at the middle of the clamped edges.

$b<a$

\begin{tabular}{|l|l|l|}
\hline \multirow{4}{*}{$a / b$} & \multicolumn{2}{|l|}{$M_{y y}=\beta_{3} p_{0} b^{2}$} \\
\cline { 2 - 3 } & Present study & $\begin{array}{l}\text { Timoshenko and Woinowsky-Krieger } \\
{[10]}\end{array}$ \\
\hline 1.1 & -0.2956 & -0.2956 \\
\hline 1.2 & -0.3084 & -0.3084 \\
\hline 1.3 & -0.3176 & -0.3176 \\
\hline 1.4 & -0.3240 & -0.3240 \\
\hline 1.5 & -0.3288 & -0.3288 \\
\hline 2 & -0.3368 & -0.3368 \\
\hline
\end{tabular}


Galerkin-Vlasov Method for the Flexural Analysis of Rectangular Kirchhoff Plates with Clamped ..

\begin{tabular}{|c|c|c|c|}
\hline & $\infty$ & -0.3332 & -0.3332 \\
\hline \multicolumn{4}{|l|}{$b>a$} \\
\hline & \multirow{2}{*}{$b / a$} & \multicolumn{2}{|c|}{$M_{y y}=\beta_{3} p_{0} b^{2}$} \\
\hline & & Present study & $\begin{array}{l}\text { Timoshenko and Woinowsky-Krieger } \\
{[10]}\end{array}$ \\
\hline & 1. & -0.2788 & -0.2788 \\
\hline & 1.1 & -0.3148 & -0.3148 \\
\hline & 1.2 & -0.3472 & -0.3472 \\
\hline & 1.3 & -0.3752 & -0.3752 \\
\hline & 1.4 & -0.3992 & -0.3992 \\
\hline & 1.5 & -0.4196 & -0.4196 \\
\hline & 1.6 & -0.4360 & -0.4360 \\
\hline & 1.7 & -0.4488 & -0.4488 \\
\hline & 1.8 & -0.4608 & -0.4608 \\
\hline & 1.9 & -0.4696 & -0.4696 \\
\hline & 2 & -0.4764 & -0.4764 \\
\hline & 3 & -0.4984 & -0.4984 \\
\hline & $\infty$ & -0.50 & -0.50 \\
\hline
\end{tabular}

\section{Discussion of Results}

The Galerkin-Vlasov variational method has been applied in this work to determine the deflection function and deflection at the centre of a rectangular Kirchhoff plate with two opposite edges $(y= \pm b)$ clamped and the other opposite edges $(x=0, x=2 a)$ simply supported, for uniformly distributed load on the entire plate region. The Galerkin-Vlasov variational functional constructed using the product of the eigen functions of vibrating simply supported Euler - Bernoulli beams (in the $x$-direction) and the eigen functions of clamped clamped Euler - Bernoulli beams (in the $y$-direction) is given in Equation (26). The Garlerkin-Vlasov variational functional is expressed in general in terms of undetermined displacement parameters $w_{m n}$ of the displacement function $W(x, y)$.

Solving with the aid of the online Mathematica integration software gave the undetermined displacement parameter $w_{m n}$ given in Equation (39) and the displacement function given in Equation (45). The maximum displacement of the plate was found to occur at the centre of the plate, in line with the symmetrical features of the plate and the loading, and is tabulated as Table 1. A comparison of the maximum deflection of the SCSC thin plate with the Timoshenko results shows that for $m=1, n=1$, a relative percentage difference exists ranging from $1.39 \%$ for plates with aspect ratios of infinity to about $4.5 \%$ to square plates. The difference between the present study and the Timoshenko results reduced significantly to an average of $-0.37 \%$ for $m=1$, $3, n=1$, showing the rapidly convergent property of the deflection function Equation (45) obtained in this study and showing good agreement with the Timoshenko and Woinowsky's results obtained using the principle of superposition of solutions for simply supported plates and solutions for plates carrying edge bending moments.

Tables 2 and 3 present the converged maximum deflection solutions in terms of the plate aspect ratios obtained in the present study, and compare them with solutions obtained by Timoshenko and WoinowskyKrieger [10] for the two cases when $b<a$, and when $b>a$. The tables illustrate the excellent agreement between the two results for maximum deflection at convergence of the Galerkin-Vlasov solutions. Tables 4 and 5 presents a convergence study of the behaviour of the series for maximum deflection function (for $b\langle a$, and $b\rangle$ a) for various aspect ratios. The tables show that convergence is achieved using five terms of $m$, and $n$ i.e. $m=1$, $3,5,7,9$ and $n=1,2,3,4,5$.

Tables 6 and 7 present the bending moment coefficients for $b<a$ and for $b>a$ and for various plate aspect ratios at the center of the plate. The tables illustrate the excellent agreement between the present study and the results from Timoshenko and Woinowsky-Krieger [10]. Table 8 presents the bending moment coefficients at the middle of the clamped edge for $b<a$ and for $b>a$ for different plate aspect ratios; and compares the results with the Timoshenko and Woinowsky-Krieger results. The table shows excellent agreement of the present study with the Timoshenko and Woinowsky-Krieger results.

\section{Conclusions}

The Galerkin-Vlasov variational method has been applied in this study to determine the deflection function and the maximum deflection of a rectangular Kirchhoff plate with two opposite clamped edges $(y= \pm b)$ and the other two opposite edges $(x=0, x=2 a)$ simply supported. The plate studied was considered isotropic, homogeneous, thin, and under small deflections and subject to a uniformly distributed load of intensity $p_{0}$ on the entire plate domain.

The displacement shape functions were found using the Vlasov model as the product of the eigenfunctions of a clamped clamped Euler - Bernoulli beam in the corresponding clamped direction and the eigen- 
functions of a simply supported Euler - Bernoulli beam in the corresponding simply supported direction. The plate variational functional was then derived using Galerkin-Vlasov methodology and solved to obtain the unknown parameters of the displacement field, and hence the displacement field.

The maximum deflection and maximum bending moments were found to occur at the centre of the plate, satisfying the requirements of symmetry of the plate and the loading. In order to verify and validate the results of this study, numerical values of the maximum deflection and maximum bending moments were obtained for various plate aspect ratios, and compared with the Timoshenko and Woinowsky solution of the same problem. The present results show good agreement with the classical solution obtained by Timoshenko and Woinowsky. The effectiveness of the Galerkin-Vlasov method is thus illustrated. The results obtained for both the deflections and bending moments were exact solutions.

(i) The Galerkin-Vlasov method yielded rapidly convergent double series for the deflection function for the Kirchhoff plate with opposite edges simply supported and the other edges clamped for the case of uniformly distributed load over the entire plate surface.

(ii) The deflection functions obtained converged to the exact solution for $m=1,3,5,7,9 ; n=1,2,3,4,5$. However, satisfactorily accurate results were obtained for $m=1,3 ; n=1,2$.

(iii) The expressions obtained for bending moments were also rapidly convergent double series, and satisfactory results with marginal relative error were obtained using a few terms of the series.

(iv) Galerkin-Vlasov method gave closed form analytical solutions to the Kirchhoff plate problem considered in this study.

\section{References}

[1] Sebastian V.K. (1983): An elastic solution for simply supported rectangular plates. Nigerian Journal of Technology, NIJOTECH Vol.7 No1, Sept, 1983 pp 11-16.

[2] Ugural, A.C. (1999): Stresses in Plates and Shells McGraw Hill Book Co. New York.

[3] Szilard, R. (2004): Theories and Applications of Plate Analysis: Classical, Numerical and Engineering Methods. John Wiley \& Sons Inc, New Jersey.

[4] Ventsel, E. and Krauthammer T. (2001): Thin Plates and Shells: Theory, Analysis and Applications Marcel Dekker Inc. New York USA.

[5] Reissner E. (1945): The effect of shear deformation on the bending of elastic plates. Journal of Applied Mechanics, Vol 12 pp A69.

[6] Mindlin, R.D. (1951): Influence of Rotary Inertia on Flexural Motion of Isotropic, Elastic Plates. Journal of Applied Mechanics vol. 18 No 1 pp. $31-38$

[7] Mindlin, R.D. and Deresiewicz H. (1954): Thickness Shear and Flexural Vibrations of a Circular Disk. Journal of Applied Physics 25 (10) pp $1329-1332$.

[8] Mindlin, R.D., A. Schacknow and H. Deresiewicz (1956): Flexural Vibrations of rectangular plates. Journal of Applied Mechanics vol 23 no 3 pp 430 - 436 (view Google Scholar)

[9] Chandrashekhara, K. (2011): Theory of plates. Universities Press (India) Limited Hyderabad p269 - 275.

[10] Timoshenko, S.P. and Woinowsky-Krieger S. (1959): Theory of Plates and Shells Second Edition. McGraw Hill. 580p.

[11] Navier C.L.M.N. (1823): Bulletin des sciences de la societie philomatique de Paris 5, pp 95-102, Extrait des recherché sur la flexion des planes elastiques.

[12] Levy M. (1899): Memoire sur la theorie des plaques elastiques planes. Journal de mathematiques pures et Appliquée Vol. 30 pp. 219-306.

[13] Osadebe N.N., Ike C.C., Onah H., Nwoji C.U. and Okafor F.O. (2016). Application of the Galerkin-Vlasov method to the flexural Analysis of Simply Supported Rectangular Kirchhoff plates under Uniform loads. Nigerian Journal of Technology NIJOTECH, Vol 35 No 4, October 2016 pp. 732-738.

[14] Aginam C.H., Chidolue C.A., and Ezeagu C.A. (2012): Application of Direct Variational Method in the Analysis of Isotropic thin Rectangular Plates. ARPN Journal of Engineering and Applied Sciences Vol.7, No 9, September 2012, pp. 1128-1138, Asian Research Publishing Network (ARPN) www.arpnjournals.com

[15] Mbakogu F.C. and Pavlovic M.N. (2000): Bending of clamped orthotropic rectangular plates: a variational symbolic solution. Computers and structures 77, (2000) pp. 117-128 Permagon.

[16] Reddy J.N. (2002). Energy Principles and Variational Methods in Applied Mechanics. John Wiley and Co. Inc. New Jersey, USA

[17] Balasubramanian Ashwin (2011): Plate Analysis with Different Geometries and Arbitrary Boundary Conditions. MSc Thesis Mechanical Engineering, Faculty of Graduate School, the University of Texas at Arlington, December 2011.

[18] Kantorovich L.V. and Krylov V.L. (1954): Approximate methods of Higher Analysis. John Wiley and Sons, New York.

[19] Ezeh J.C., Ibearugbulem O.M. and Onyechere C.I. (2013). Pure Bending Analysis of thin Rectangular Flat Plates using ordinary finite Difference Method. International Journal of Emerging Technology and Advanced Engineering, Vol. 3, Issue 3, March 2013 pp. 20-23. 